Archives de sciences sociales des religions

123 | juillet - septembre 2003

Varia

\title{
Lilith : une androgynie oubliée
}

\section{Vanessa Rousseau}

\section{OpenEdition}

Journals

Édition électronique

URL : http://journals.openedition.org/assr/1067

DOI : $10.4000 /$ assr. 1067

ISSN : $1777-5825$

Éditeur

Éditions de l'EHESS

Édition imprimée

Date de publication : 1 juillet 2003

Pagination : 61-75

ISBN : 2-222-96737-6

ISSN : 0335-5985

Référence électronique

Vanessa Rousseau, "Lilith : une androgynie oubliée », Archives de sciences sociales des religions [En ligne], 123 | juillet - septembre 2003, mis en ligne le 17 novembre 2005, consulté le 19 avril 2019. URL : http://journals.openedition.org/assr/1067 ; DOI : 10.4000/assr.1067 


\section{LILITH : UNE ANDROGYNIE OUBLIÉE}

Lilith est peu connue, et pour cause...oubliée par la chrétienté depuis plusieurs siècles, gardée à l'esprit par les croyances juives bien que pourtant évitée, cette figure féminine issue des différents récits de la Genèse ne trouva que très peu de place dans les mémoires.

Dans les communautés juives traditionnelles, la croyance portée à Lilith, première compagne d'Adam, persista jusqu'à la seconde moitié du XIX siècle, période au cours de laquelle de nombreuses pratiques liées à la démone trouvèrent leur place. C'est à compter de ce siècle que Lilith entre dans l'oubli pour finalement refaire surface dans les esprits et les écrits à compter de la seconde moitié du $\mathrm{XX}^{\mathrm{e}}$ siècle. Ce n'est en effet qu'à partir des années mille neuf cent soixante dix que son "visage » et sa personnalité réapparaissent dans des récits poétiques, des fíctions et des créations littéraires semble-t-il influencées par les mouvements de libération de la femme nés à cette époque. Adoptée par des féministes juives américaines comme symbole de l'indépendance des femmes, Lilith devint également un objet de recherche pour les théologiens et historiens des doctrines religieuses soucieux d'une possible judéo-chrétienté des mythes et croyances issus des Saintes Écritures. Nous trouvons de nos jours sur internet de nombreuses adresses répondant au nom de Lilith où de nombreux sites la présentent comme le symbole de la lune noire astrologique. Elle correspondrait, selon ces sites, non pas à une planète ou à un astre, mais à un point sensible particulier situé sur l'orbite elliptique de la lune en rapport avec la terre : un des obscurs foyers de l'orbite lunaire. Ce lien étroit entre l'astrologie et la figure mythologique de Lilith trouve sa justification dans les croyances passées attachées à cette démone primitive. C'est donc en faisant appel à la magie, à la divination et à l'astrologie que son visage pourrait être mis à jour. Edouard Dhorme, historien des religions, démontre dans ses ouvrages en quoi les Babyloniens, pères de la tradition hébraïque et héritiers de la science suméro-akkadienne, influencèrent l'enthousiasme accordé à l'astrologie dans les mythes assyro-babyloniens (1). Lilith a gardé en elle la trace d'un tel intérêt, et chose surprenante, nous trouvons jusqu'à nos jours les traces de cette origine astrale. Il y a donc sur nos écrans d'ordinateurs des croyances qui dérivent des

(1) Édouard Dhorme, Religions de Babylonie et d'Assyrie, tome II, chap. X, Paris, PUF, 1949, p. 266. (coll. «Mana»). 
quelques millénaires antérieurs. Mais Lilith est aussi sur internet lamfigure féminine la plus représentative des mouvements lesbiens, l'égérie de la création picturale, le personnage fétiche de quelques bandes dessinées Manga qui présentent cette femme avec des atouts physiques accrocheurs, à la fois ronds et pointus, représentatifs de la douceur maternelle et cependant empreints d'une force et d'une violence dignes d'un mâle.

La psychologie moderne prête actuellement un grand intérêt à cette femme démoniaque. Reconnue aujourd'hui comme mère des tziganes, Lilith ne se cantonne pourtant pas à cette communauté, elle hante nos systèmes de représentations, à mi-chemin entre le politique, l'esthétique et le sexuel.

\section{Une femme primordiale qui épouse l'enfer}

Lilith est considérée dans la tradition juive comme la première épouse d'Adam. Née comme lui du limon de la terre elle fut remplacée par Ève après avoir refusé de se soumettre à l'autorité de son époux. Réputée avoir été par la suite la compagne de Sammaël, cette femme primordiale est également dans la tradition islamique l'épouse du diable avec qui elle dormit et donna naissance aux Jinn (Jinni). Dans les légendes islamiques postérieures au Moyen Âge, elle est devenue un succube, un démon et une divinité de l'onanisme qui donne naissance à des démons nommés, de par leur origine, des Lilims.

Le folklore juif, quant à lui, présente Lilith comme une démone ennemie des nouveau-nés. L'Alphabet de Ben Sira, premier conte à développer sa mythologie, écrit entre le VII et le $\mathrm{X}^{\mathrm{e}}$ siècle, témoigne de l'histoire de Lilith, de sa fuite du paradis pour ne pas avoir voulu se soumettre aux volontés d'Adam, et de ses nombreux infanticides. Présente dans la majeure partie du corpus de la littérature juive, Talmud, Midrashim, Kabbale ou folklore populaire, Lilith apparaît toujours comme un démon nocturne et néfaste qui concentre en elle la mort (2). Oubliée par le christianisme, bien que présente en Isaïe au livre XXXIV (verset 14), les rares ouvrages du Moyen Âge et de l'Époque moderne qui la citent ne font référence à elle qu'à titre de superstition et de croyance juive désuète voire insensée. Saint Augustin (354-430) relève l'audace et le manque de raison de ceux qui accordent à cette femme la moindre existence (3) et il faut attendre le $\mathrm{XVI}^{\mathrm{e}}$ siècle, période au cours

(2) Les sources hébraïques utilisées sont pour les Midrashim et le Talmud: Midrash ber'eshit Rabba [BR], Éditions Vilna, 1887 (cité par chap. et par §.) / le Midrash Tanhuma' Haqadum weha-yashan [Tan B], Editions Buber, Vilna, 1885 (cité par livre et par page) / Genèse Rabba, Édition critique de J. Théodore et C. Albeck, Berlin, 1912-1927 (traduction française de M. Stern, 1981-1985) / Nombres Rabba [Num Rab.], Éditions Vilna, 1884 / Midrash Abkir (Midrash compilé au IX siècle, aujourd'hui perdu), certains passages sont cités dans le Yalkut Shimoni, le Yalkut Reubeni et le Séfer Yalkut Re'ubeni "al ha-Torah », Paris, Fayard, 1987 / Talmud de Babylone, Lagrasse-Verdier, 1986 (les abréviations utilisées pour les passages concernés du Traité seront les suivantes : B. Erubin et B. Sanhédrin). Un dernier ouvrage : L'Avot dé-Rabbi Nathan, Vienne, Salomon Schechter, 1887, reproduction anastaltique : New-York, 1945.

(3) Augustin, Contre les adversaires de la Loi et des Prophètes, livre II, chap.1, § 2, tome XXXVI, Paris, Édition Louis Vivès, 1878. 
de laquelle se succèderont les nombreuses publications de dictionnaires bibliques pour trouver d'autres traces de Lilith dans les écrits d'exégèse chrétiens.

Le texte le plus représentatif de cette présence date du XVIII ${ }^{\mathrm{e}}$ siècle et pourrait être une synthèse éclairante des textes antérieurs qui ont tenté de définir cette femme obscure. Il s'agit de l'article Lilith du dictionnaire historique de la Bible publié par l'exégète et historien Augustin Calmet (1672-1757) en 1722 :

«Lilith étoit, disent les rabbins, la première femme d'Adam qui se sépara de son mari ; et ne voulut plus retourner avec lui, quoique Dieu lui eût envoyé deux anges pour l'y contraindre. Ils croyent qu'elle mange les enfans nouveau-nez. C'est pourquoi les juifs, lorsqu'un enfant est né dans une maison écrivent avec de la craye ou autrement qu'Adam et Ève soient ici, que Lilith s'en éloigne. Ils écrivent aussi les noms des trois anges qui poursuivent Lilith ; sçavoir : Sennö̈, Sansennö, Samangeloph; parce que Lilith leur promit de ne faire aucun mal aux lieux où elle trouvoit leurs noms. Nous avons déjà parlé de Lilith sous l'article de Lamia. Isaïe (XXXIV.14) fait mention de Lilith et saint Jérôme la traduit par Lamia, et les Septante par Onocentaure. Nous croyons que ce terme signifie un oiseau nocturne, et de mauvaise augure, comme la choüette, le hibou, le chat huant, la chauve-souris. Lilith en hébreu signifie la nuit » (4).

C'est là le meilleur exemple des connaissances sur Lilith dont les exégètes chrétiens de l'Époque moderne disposaient. Il n'y a guère de variantes, et Augustin Calmet semble réunir toutes les données et propos issus de la tradition chrétienne la concernant. Aucune allusion n'est faite à son caractère androgyne, seule sa cruauté et son aspect dévorateur sont présents. C'est dans les traités de superstitions et de croyances que nous trouvons également de tels propos qui, lorsqu'ils ne rattachent pas spécifiquement Lilith à la tradition juive, ce qui est le plus souvent le cas, qualifient la croyance en cette figure féminine d'attitude judaïque sans fondements.

Si la tradition juive est d'accord avec les descriptions et narrations présentes chez Augustin Calmet, elle comporte pourtant une variante essentielle quant au caractère androgyne de cette femme primordiale ; qui dans le cas du judaïsme n'est pas Ève, mais Lilith, laquelle serait porteuse d'une sexualité ambiguë. Cela étant, on peut dire que Lilith touche des thèmes qui s'attachent aux croyances et aux conceptions que le judaïsme et le christianisme se font de la sexualité et de l'incorporation alimentaire.

\section{Fractures amorcées entre judaïsme et christianisme}

L'étude du rapport de filiation entre judaïsme et christianisme révèle l'importance prise par les anaphores, les images et les systèmes de représentations relatifs au sexuel et à l'alimentaire dans l'élaboration croyante des législations religieuses qui structurent le monde.

(4) Augustin CALmet, Dictionnaire historique, critique, chronologique, géographique et littéral de la Bible, Paris, Emery, Saugrain et Pierre Martin, 1722, tome 1, p. 484. 
Durant les dix premiers siècles d'expansion de la foi chrétienne, le lien le plus manifeste entre l'Ancienne et la Nouvelle Alliance se trouve être étroitement attaché aux questions alimentaires et à la fornication (5). Chères à ces deux phénomènes liés à la reproduction de l'espèce, les substances telles que le sang, le lait, la graisse et le sperme nourrissent sans répit les actes constitutifs du cosmos chrétien et de son gouvernement spirituel. Dès le récit du péché originel, c'est un symbolisme intégrant l'incorporation alimentaire qui se dessine, engendrant par l'ingestion d'un fruit défendu, la naissance de la sexualité. Au cours de ce récit la mort et la naissance apparaissent simultanément : le péché accompli, le sang sera amené à couler, de même que le sperme.

Lilith concentre en elle toutes les craintes et les terreurs que le mystère de la procréation fait naître ; elle résulte donc d'une tradition judaïque dont les différentes légendes trouveront traces dans la tradition chrétienne; ses éléments majeurs seront le sang et son équivalent symbolique, le sperme. Créature monstrueuse suceuse de sang et dévoreuse de sperme (6), toujours ralliée au féminin par son statut de mère des Démons (7) et profanatrice de la sainte semence humaine, elle est symboliquement présente dans la plupart des écrits pré-bibliques.

Lilith est bannie des Saintes Écritures : sa seule apparition (8) la présente sous un nom d'emprunt en Isaïe (XXXIV.14). Elle est dans la tradition hébraïque la profanatrice du bon usage de la force génératrice, celle qui favorise l'émission de la

(5) De nombreux interdits juifs ont été préservés par la chrétienté occidentale durant les premiers siècles de notre ère. Tel est le cas de l'interdit des viandes suffoquées, du sang et de la fornication. Cet interdit fut préservé pendant les huit premiers siècles. Travaillant sur le sang-aliment, qui est l'objet de notre thèse, nous avons constaté par la lecture des différents textes patristiques que la tradition juive est un germe et une puissance formatrice des représentations, voire des pratiques, chrétiennes durant les dix premiers siècles de la chrétienté. Une constatation qui permet de concevoir les mythes présents dans les Saintes Écritures comme des sphères communes aux juifs et aux chrétiens que le concept de judéochristianisme englobe et traduit.

(6) Concernant les relations de Lilith au sperme : Gershom Scholem, La Mystique Juive, chap. Shekina (les facteurs passifs et féminins de la divinité), Paris, Cerf (traduction de Maurice R. Hayoum), (coll. «Patrimoines du Judaïsme »/Concernant la parenté de Lilith avec les Lamies et l'Empousa gréco-latine : Jacques BriL, Lilith ou la mère obscure, Paris, Payot, 1981, p. 76.

(7) Lilith est une dévoreuse de sperme incitant l'homme par ses séductions nocturnes à l'onanisme. De cette procréation démoniaque sont façonnés des démons : la semence expulsée à terre ou déversée dans l'antre buccale de Lilith engendre des monstres tels la Mandragore (fille issue du sperme des pendus et de la terre réceptrice de la semence). Sur ces phénomènes et leurs variantes cf. Gershom ScholEm, La Kabbale et sa symbolique, Paris, Payot, 1966, pp. 171 et ss. (traduit de l'Allemand par Jean Boesse).

(8) Ce statut d'unique passage est pourtant à relativiser, car nombreuses sont les allusions à la démone... notamment en Esaïe XIII.21 et en Job XVIIII.17-21 (métaphore de la Misère). Relevons également que les différentes versions de la Septante ainsi que de nombreuses versions de la Vulgate traduisent le plus souvent Lilith par Lamia (d'origine grecque), ce qui relance la difficulté de discerner le domaine d'existence de cette démone réputée adroite pour se travestir et s'infiltrer dès que possible dans le domaine d'Ève ou de l'épouse "légitime ». La version française des Septante fournie par la Bible polyglotte de F. Vigouroux, Paris, Éditions A. Roger et F. Chernoviz, 1902, emploie ainsi le terme de Lamia plutôt que celui de Lilith, non seulement pour les versions grecques des Saintes Écritures, mais également pour celles de la Vulgate. F. Vigouroux signale que dans les versions hébraïques le mot hébreu correspondant peut non seulement signifier Lilith, mais également chat-huant (les termes de chouette ululante et d'empuse sont de même souvent employés par les versions grecques). Concernant les polémiques sur la présence ou absence de Lilith dans les textes Saints : cf. Augustin, op . cit. 
semence hors de toute union charnelle. Ce sperme émis hors de la voie vaginale devient alors la substance qui façonne les démons, au dépens des enfants qui ne pourront pas naître. Cette femelle vierge, et pourtant mère des démons (9), fait du sperme son ambroisie, elle le façonne comme Dieu la façonna à partir de la terre, elle l'incorpore par les voies buccale et anale en gonflant ses antres de cette semence.

Les nombreux passages de la tradition hébraïque qui font mention de Lilith la présentent également comme un animal sauvage suceur de sang dont l'anatomie est de nature ophidienne et tellurique. Ces données laissent la première compagne d'Adam concentrer en elle tout l'envers symbolique d'Ève, son envers mais également son complément masculin : telle une version du couple originel où le sperme, concentré par le féminin, serait dépouillé de toute fonction de procréation (la fécondation y étant buccale).

S'intéresser à l'existence oubliée de Lilith est donc tenter de comprendre le schème de la reproduction et de la distinction des sexes dans les différents récits du péché originel. Quant à ses dérivés mythologiques, dont l'origine supposée serait suméro-babylonienne (10), ils ne sont pas sans rapport avec le thème de la consommation du sang (11); consommation à l'origine des nombreuses prohibitions alimentaires et interdits sexuels que la chrétienté occidentale édictera durant de nombreux siècles.

En s'attachant aux actes d'incorporations alimentaire et sexuelle, Lilith concentre en elle tous les démons de l'analogie. Son contentement, inépuisable, relevant des deux substances, le sang et le sperme (12), fait des sphères érotiques et reproductives les domaines et les éléments nourriciers de ses perpétuels désirs de renouvellement.

L'Histoire de Lilith passe par sa création; mais avant d'évoquer son statut de première femme, compagne de l'Homme primordial, reprenons le sens et le choix de l'Ancien Testament. Commençons par l'acte fondateur de notre histoire: la "chute de l'homme », celui où s'exprime le génie démoniaque de Lilith, une femme qui travestit ses charmes et fait de sa bouche l'instrument d'un péché véniel.

\section{Les différentes versions de la genèse}

Les versions juives de la création d'une femme primordiale, distinctes des versions chrétiennes, s'attachent à l'hypothèse d'une androgynie divine. Le caractère

(9) Il serait plus qu'intéressant de travailler à la signification de la virginité de Lilith au regard de celle de Marie (l'une contenant en elle le fruit de ce que l'autre a reçu [la semence] sans enfanter).

(10) Sur les origines mythologiques et linguistiques de Lilith, cf. Jacques BRIL, op. cit., chap. II, p. 53. Jacques Bril voit dans le couple d'Ardat Lili et de Lilû (divinités dérivées du Lil sumérien) les formes archaïques de la Lilith juive. Ardat Lili, vierge inassouvie attaque en effet les hommes mariés pour sucer leur sang et leur semence.

(11) Ce sujet est l'objet de notre thèse sous la direction de Robert Muchembled intitulée : "Le goût du sang ; Pratiques, représentations et polémiques dans la chrétienté occidentale à l'Âge moderne » (soutenance en novembre 2003).

(12) Deux substances qu'elle est réputée dérober aux hommes lors des rapports sexuels (elle prend ainsi selon les versions le visage d'une lamie, "vampire » au féminin). 
androgyne de l'homme primordial créé à l'image du Dieu est largement présent dans le corpus juif, mais omis par la tradition chrétienne. On peut noter cette divergence de récits dans les passages tirés de la Genèse aux livres I et II. Une combinaison des versions a suscité des contradictions difficiles à résoudre pour les théologiens hors du contexte historique dans lequel ces divergences de foi prennent leur source. On trouve ici la trace de la rencontre de deux croyances et de deux traditions: l'une juive et sacerdotale; l'autre de souche chrétienne. Concernant l'androgynie de l'homme primordial, il est tentant de rapprocher les traditions hébraïque et babylonienne, même si les influences assyro-babylonienne et sumérienne sur les écrits du Pentateuque ne doivent pas être considérées comme uniques; la tradition grecque est également riche de nombreux mythes liés à l'androgynie tant humaine que divine. Il s'agit simplement de mentionner l'oubli par les versions chrétiennes de la Genèse des diverses figures de cet homme primordial et de ses avatars. Concernant notre Lilith, il suffit de relever que son origine semble issue des récits assyro-babyloniens de l'épopée du dieu Gilgamesh. On trouve en effet dans ce récit les traces d'un $L i l$ sumérien en rapport étroit avec le Dieu Enkidu, une divinité androgyne primordiale aux ressources multiples parmi lesquelles la tradition juive a pu puiser en partie ses propres sources et représentations. Comme de nombreux autres personnages de la tradition hébraïque, Lilith n'est pas juive à l'origine, elle trouve sa source dans la mythologie mésopotamienne. Femme aux formes sexuelles exagérées, elle est représentée avec un ventre énorme renfermant des torrents de sperme. Telle la Vénus gourmande du sperme d'Adonis, Lilith est une mère à la fois dévoreuse et pourvoyeuse qui accumule ce dont elle se nourrit jusqu'à l'excès. Dans le panthéon babylonien, un démon du nom de Lilîu, hypostase d'un Lil sumérien antérieur (13), séduisait les femmes pendant leur sommeil. Lilith, descendant probable de ce démon, est une vierge inassouvie qui ne s'est jamais dévêtue et dont les mamelles sont toujours restées vides. Il faut relever ici que le premier récit de la Genèse est né peu après le retour des Hébreux de l'exil babylonien. De nombreuses « déesses-mères » babyloniennes, androgynes, avaient été adoptées alors et étaient encore vénérées par les Hébreux. La Babylonie apparaît donc influente dans la création des récits de la Genèse : une riche « collection » de légendes (14).

Ces caractéristiques appuient l'hypothèse d'une Lilith vierge mais prostituée, qui a des relations sexuelles sans pourtant mener le coït à son terme. Dévorant le sperme des hommes jusqu'à leur total épuisement, elle est de plus ravisseuse et dévoreuse d'enfants, ce qui laisse supposer qu'elle étouffe toutes les forces reproductrices jusqu'à les rendre stériles. Lilith pousse les hommes à des « consommations improductives » de leur énergie. Dans la Bible, Onan est foudroyé par le Seigneur parce qu'il ne répand pas sa semence in vas naturale. Qui pourrait, sinon Lilith, susciter de telles manifestations?

(13) Cf. Robert Graves et Raphaël Patai, Les Mythes Hébreux, Paris, Fayard, 1987, note 5, p. 85 : " On fait d'ordinaire dériver Lilith du mot assyro-babylonien Lilitû, démon femelle ou Esprit du vent (...) Mais elle apparaît antérieurement sous la forme Lillake sur une tablette d'UR (2000 ans av. J.C.) qui contient le conte de Gilgamesh et le Saule. »

(14) Sur le lien étroit entre mythes assyro-babyloniens et mythes hébreux, cf. Édouard DHORME, "Les Religions de Babylonie et d'Assyrie" in Les anciennes religions orientales, Paris, PUF, 1949, (coll. « Mana ») et Jean Bottero, La Religion Babylonienne, Paris, PUF, 1952. 
En faisant de sa bouche l'antre de tous les désirs, la démone est également accusée d'inciter à une sorte de "fécondation buccale ", phénomène inadmissible qui sous-entendrait que l'acte sexuel aurait d'autres fins que la simple reproduction de l'espèce, ce que condamnent les Saintes Écritures du fait de l'utilisation stérile et à contre-emploi de la sécrétion destinée à la reproduction. La semence doit en tout lieu et en tout temps être conservée du dedans (fécondée et nourrie de l'intérieur) : d'ailleurs, la semence qui ne se répand pas in vas naturale change de statut et devient excrément.

Le fait que la racine babylonienne lil évoque la terre, racine que l'on retrouve dans le nom du Dieu Enlil (15), explique que Lilith soit représentée comme un démon de la terre associant forces nocturnes (16) et forces génitrices, les premières étant sans destination productive, les secondes étant porteuses de la série des êtres à venir. Le fait qu'elle soit en mesure de recevoir la semence tout en la sécrétant révèle son caractère androgyne. Ne recevant pas la semence par voie vaginale mais par voie buccale, Lilith consume ainsi les forces génitrices de ses pères sans pour autant être en mesure de procréer (l'estomac reçoit ce que l'utérus aurait dû conserver pour une maturation possible du fruit de l'acte sexuel).

\section{La création de la femme : un souci d'identité}

Dans les Saintes Écritures, la femme naît d'un double «constat »: de même chair qu'Adam, elle en est pourtant séparée, et sitôt l'interdit posé, elle est en mesure de le tenter. Ces éléments suggèrent que la distinction fondamentale entre l'homme et son Dieu s'incarne dans l'apparition de la sexualité. Les traditions autour de la création d'Ève sont multiples, encore faut-il entendre par ce nom d'Ève la première compagne d'Adam. La difficulté pour retracer l'existence de ce premier 'adâm femelle résulte pour une part de la coexistence dans la Genèse de deux récits distincts concernant la création de l'homme. Cette difficulté n'est pas atténuée par les traditions hébraïques parfois très distinctes du fonds chrétien.

Nous trouvons dans la Genèse (17) la création simultanée, le sixième jour, de l' 'adâm mâle et de l' 'adâm femelle. Qui était donc cette femelle, puisque le récit de la création d'Ève n'est pas encore sensé avoir trouvé naissance? Suivant les traductions, nous trouvons que Dieu créa l'homme à son image, c'est-à-dire qu'il « les créa mâle et femelle... ». Cette confusion du singulier et du pluriel rend ce récit plus que troublant en mettant à jour une androgynie supposée du divin. L'explication trouve pourtant sens si nous n'omettons pas de relever qu'avant la chute, le monde du Paradis était un monde du non-partage et de la non-distinction tel une

(15) Dans la religion assyro-babylonienne, héritière de la mythologie mésopotamienne du $\mathrm{VII}^{\mathrm{e}}$ millénaire AEC, les forces divines sont forces de la Nature et chaque divinité est gardienne de son territoire. Le dieu Enlil était considéré aux VIII et VII ${ }^{\mathrm{e}}$ siècles avant J.C. comme seigneur de l'Atmosphère, le créateur de l'univers, dieu de la végétation et de l'Ouragan qu'il utilise comme arme de combat que l'on nomme Amarou ou Déluge. Il faut cependant noter que la première apparition de Lilith sous la forme de Lilithu trouva naissance dans l'Épopée de Gilgamesh, roi de la cité sumérienne légendaire nommée Uruk.

(16) Lilith est apparentée à la nuit, dont l'hébreu lä̈l (qui signifie «nuit ») peut justifier le rapprochement d'un point de vue linguistique et symbolique.

(17) Genèse I.27 : «Dieu créa l'homme à son image, à l'image de Dieu il les créa ; mâle et femelle il les créa. » (version de la TOB, Alliance biblique universelle, Paris, Cerf, 1988). 
unité primordiale dépourvue d'accidents. Le troisième livre de la Genèse est le plus souvent cité parce que semble t-il le moins inquiétant: Adam est créé à partir de glaise... (18) et Dieu tire ensuite de sa côte une femme afin de lui éviter la solitude (Gen. II.18-23). Ce déroulement est plus « commode » pour entretenir l'idée d'une primauté du masculin sur le féminin malgré le fait que l'androgynie d'Adam puisse persister par l'image d'une féminité, arrachée de son corps pour être posée « en face de lui ».

Gardons pour l'instant présent à l'esprit les contradictions que ces deux récits peuvent engendrer en relevant les moyens par lesquels la tradition chrétienne, mais également la juive, ont fait de cette opposition le fondement conceptuel de la différenciation des sexes.

Voyons donc les quelques versions les plus communément admises: Mâle et femelle il les créa, il les bénit, et il les appela du nom d'Adam (19). Il les créa ainsi à l'état non séparé, justifiant du jeu de mot entre 'ishshâh (femme) et 'ish (homme). Lorsque Adam fut placé dans le jardin d'Eden et qu'il reçut la Loi, il lui manquait une composante : la sexualité. L'Adam créé, à la fois homme et femme (20), n'effectua le passage de la virtualité à la réalité, de l'être androgyne en son principe à un couple sexuellement différencié, qu'au terme d'un troisième geste créateur.

Dieu, constatant qu'il n'est pas bon que l'homme soit seul lui présente tous les animaux puis « anime » Ève en la tirant de sa côte. S'il n'est pas bon que l'homme soit seul c'est qu'une « aide en face » (ou « aide contre ») est nécessaire. Mais c'est aussi parce que l'unité de l'homme pourrait l'inciter à se considérer lui-même comme être divin... c'est donc la femme qui rappelle à l'homme son historicité (un c'est Dieu, deux c'est l'homme). Dans l'esprit du judaïsme, c'est Lilith qui fut d'abord donnée à Adam pour combler sa solitude. Différents recueils de Midrashim (21) la présentent comme fabriquée d'immondices et de boue, d'autres la proposent comme tirée du limon de la terre (22). Ces deux visions de la création présentent invariablement la Femme comme créée indépendamment d'Adam et donc comme nullement tirée de la chair de cet homme primordial (Adam n'aurait ainsi nullement « enfanté » la première Ève). Jamais Lilith et Adam ne s'entendirent sur les manières de faire l'amour ni même sur le partage des plaisirs liés aux pratiques sexuelles. Aussi Lilith s'offusquait-elle de toujours devoir être « sous » et soumise à Adam lors de leurs rapports érotiques (23). Le fait que suivant certaines versions Lilith ait

(18) Genèse II.7 : «Le Seigneur Dieu modela l'homme avec la poussière prise du sol. Il insuffla dans ses narines l'haleine de vie et l'homme devint un être vivant. »

(19) Genèse.V.2.

(20) Sur la réversibilité des genres sexués et le caractère androgyne de l'homme primordial et de sa composante féminine : cf. Elliot.R. Wolfson, Along the Path. Studies in Kabbalistic Myth, Symbolism and Hermeneutics, chap. 2, New York, State University of New York Press, 1995, pp. 85; 166.

(21) notamment le Yalqut Reubeni ad Gen.II.21 et IV.8.

(22) Gen.Rabba.17.4.

(23) Cf., Num.Rabba (16.25) / Également présent chez Georges Frazer, Folklore in Old Testament, Testament Studies in Comparative Religion Legend and Low, tome I, chap.I, Londres, Mac Millan and co., 1919 / cf. Elliot.R. Wolfson, Circle in the Square. Studies in the Use of Gender in Kabbalistic Symbolism, chap. I, New York, State University of New York Press, 1995, p. 84 : " The union of male and female is predicated ultimately on the absorption or containment of the left side (active, merciful, overflowing male). Indeed, the negative valorization of the feminine in certain kabbalistic texts, especially the Zoharic litterature, is underscored by the fact that when the female potency is separated from the masculine the potential exists that she will evolve into a punitive or Even demonic force. Sexual coupling of male and female is indicative of an androgynous unity that has been fractured. In the ideal state, gender differentiation is neutralized and the female is absorbed back into the male. » 
été tirée d'immondices et non de terre frappait d'illégitimité sa querelle concernant la préséance des sexes. Son combat était perdu ! Lilith fut expulsée du paradis vers la Géhenne, et trouva échange équitable avec Sammaël, figure de Satan. Pourtant, Dieu envoya trois anges (24) à sa recherche, pour lui proposer un marché : si elle refusait de revenir au jardin d'Eden et de se soumettre à Adam, chaque jour que Dieu ferait un des "enfants-démons » né de sa liaison avec Sammaël mourrait. Lilith resta dans la Mer Rouge (réputée être le lieu des démons) et sacrifia ses nouveaux-nés. Mais par vengeance elle se voua au meurtre des enfants sitôt après leur mise au monde, si ce n'est dans le ventre de leur mère (25).

Adam de nouveau seul, Dieu façonna sous ses yeux une femme faite d'os, de tissus et de sécrétions animales (26). Comble ! La créature suscita chez Adam du dégoût. La seconde tentative fut donc un échec. C'est alors que selon la tradition hébraïque l'Ève tirée de la côte d'Adam vit le jour dans un état de parfait achèvement (parce que tirée d'Adam ?). Relativisons les choses: Ève tirée de la côte d'Adam est parée de bijoux et apprêtée pour le plus grand ravissement de son compagnon (27). La version de l'épisode de la côte s'avère pourtant la plus ancienne, et cela bien qu'elle apparaisse dans beaucoup d'interprétations rabbiniques en troisième place des récits justifiant la création d'Ève. La préséance d'une telle version fut semble t-il favorisée par la présentation d'un Adam possédant originellement deux faces (28), un argument sur lequel les Rabbins insistèrent, particulièrement soucieux de résoudre la contradiction entre le livre I au verset 27 et le livre II au verset 7 de la Genèse. Cette difficulté exégétique fut ainsi résolue par l'interprétation philosophique (29) d'un Adam à deux faces, à la fois mâle et femelle. Toutes les sources font état d'un premier humain créé androgyne qui fut par la suite séparé. Les interprétations restent cependant variées et plusieurs hypothèses se succèdent.

Le Traité du Talmud de Babylone (30) laisse supposer qu'à l'origine, Dieu pensa créer deux êtres humains, l'un mâle et l'autre femelle, mais qu'il préféra en composer un seul avec deux visages, l'un mâle, regardant en avant et l'autre femelle regardant en arrière (31). Après cette nouvelle tentative il changea de nouveau d'idée et supprima le visage femelle en faisant de lui un corps de femme.

Là ne s'arrêtent pourtant pas les versions. Nous trouvons en Genèse Rabba [134] l'image d'une Evve qui serait non pas tirée de la côte d'Adam mais issue

(24) Senoy, Sansenoy et Semagelof.

(25) Ceci explique qu'au XIX ${ }^{\mathrm{e}}$ siècle encore, l'on pouvait constater parmi les familles de tradition juive un rite consistant à mettre dans la chambre d'un nouveau-né une amulette contenant les noms des trois anges et une exhortation faite à Lilith de fuir la maison. p. 24.

(26) cf., Gen.Rabba 158 et 163-164 / Mid.Abkir 133-135 / B. Sanhédrin 39.A / Avot dé-rabbi Natan,

(27) cf., Gen. Rabba 161.

(28) cf., Berakot 61.a et Erubin 18.a.

(29) Déjà chez Platon, Symposium (189.d-190.d). Voir également la mythologie grecque concernant Amour, l'existence de l'androgynos (homme-femme) et du diprosopos ("à deux visages »).

(30) Cf., B. Erubin, 18a.

(31) Le lien avec la tradition gréco-latine et plus précisément la grecque concernant le caractère androgyne de l'homme à sa création est ici inévitable : cf. Luc BRISSON, Le sexe incertain. Androgynie et Hermaphrodisme dans l'Antiquité gréco-romaine, Paris, Éditions Les Belles Lettres, 1997 (coll. « Vérité des Mythes »). 
d'une prétendue queue terminée par un dard qui avait à l'origine fait partie d'Adam. Dieu coupa cette « excroissance » dont nous avons encore aujourd'hui la trace (le coccyx) et en tira Ève.

Cette version est intéressante car elle intègre tous les mythes et visages que Lilith a été en mesure de prendre : femme anguipède, tellurique (32) et bien entendu phallique (33). Si la version hébraïque dont nous venons de faire mention permet d'expliquer analogiquement les rapports de filiation entre Lilith et Mélusine, il demeure que la version la plus communément admise par toute la tradition chrétienne est celle de la côte.

Jetons un rapide coup d'œil sur les implications symboliques et conceptuelles de ce choix de l'histoire... Le mot hébreu tsalotav, pluriel du mot tséla, a été traduit par les rabbins par le mot «côté », ces derniers insistant sur la comparaison entre les deux « points de vue » de l'androgyne (deux côtés de l' 'adâm) et les deux côtés du Tabernacle. Le mot "côté » enseigne qu'il faut prendre l'extirpation de cette côte comme une limitation et délimitation du champ d'investigation humain en corps et en esprit. La femme serait ainsi frontière, limite, horizon et « aide en face de l'homme »: l'ezer kenegdo est le premier nom accordé à Ève. Cette créature tirée de lui est l'élément qui se présente «à lui devant comme identique »; telle l'advenue d'un possible monde réflexif du sujet. La femme devient reflet de ce que réfléchit l' 'adâm mâle, elle devient son «ob-jet» : ce qui donne consistance à sa forme. Avant de procéder à la création d'Ève, Dieu présente à l'homme des animaux qui s'accouplent. Au « il n'est pas bon que l'homme soit seul » succède donc ce défilé animal qui se finira par l'advenue de l'alter ego féminin. L'épreuve proposée par Dieu aux yeux d'Adam consiste donc à faire saisir à 1' adâm la distinction entre accouplement et union. C'est une sorte de rite initiatique que Dieu propose en liant l'union et l'accouplement à ce qui était consubstantiel en Adam : Ève. « L'Amour » naquit donc pour la tradition judéo-chrétienne d'un double constat: celui d'une unité originelle (même chair, même séparation) et celui (par là-même) d'une césure fondamentale génératrice d'un «face à face » dans l'union et de la recherche de ce qui manque à l'absolue symbiose du principe mâle et du principe femelle. Dans la plupart des langues, l'homme et la femme sont désignés par des racines différentes : ce qui renforce la représentation des sexes comme distincts de nature. Ainsi, il n'y aurait pas d' " 'adâm mâle » ni d' " adâm femelle » mais d'un coté l'homme et de l'autre la femme, deux corps distincts de nature cherchant dans l'accouplement le manque à l'union.

Contrairement à la représentation chrétienne de cette création, l'exégèse juive et la langue hébraïque permettent de relier substantiellement masculin et féminin en utilisant les termes 'ish et 'ishshah. En chacun de ces termes se trouve une lettre du

(32) Le Zohar stipule que le domaine d'Ève est dans les profondeurs de la mer (Zohar III.19.a). Serait-ce là une «face » mythologique cousine de la sirène gréco-latine ? Nous avons relevé que la plupart des dictionnaires hébreux modernes définissent Lilith comme une sirène prenant la forme et les attributs d'un oiseau mais également ceux d'un poisson. Cette démone aérienne, nocturne et dévorante rompt avec toutes les lois de la nature, voyage par ses débordements d'ingéniosité entre et parmi les quatre éléments primordiaux que sont l'eau, la terre, l'air et le feu. Qualifiée et reconnue par la plupart comme un animal sauvage, nous pourrions aisément retrouver son "visage » et son caractère sous les traits du serpent tentateur de la Genèse (animal entre deux mondes : un « inter-monde » en somme, entre monde des humains et univers du divin).

(33) Sur ce point et le lien d'Ève au phallique cf. Elliot.R. Wolfson, Along the Path, op. cit., p. 76. 
nom divin qui marque ainsi chaque sexe de deux substrats constitutifs de la divinité.

Si Dieu créa l'homme mâle et femelle cela laisse supposer que l'un comme l'autre, Adam comme Ève, s'intègrent à la grâce du renouvellement (restauration continue de l'image de Dieu). Mais la chose n'est pas au ciel comme sur la terre puisqu'en ce qui concerne le sexe corporel séparant l' 'adâm il en est autrement. La conscience de la femme ne peut être prise par le récit du péché originel autrement que comme conscience dérivée, puisque relative à cette existence scindée qui est en même temps et en même lieu "une ». La femme prend ainsi figure d'" atmosphère » (évanescence d'Adam) : l'élément existentiel «donné là », dont l'unité, la mémoire et la pensée sont situées dans un «quelque chose » de plus stable, dans un "support-réceptacle» de toutes ses tendances et de tous ses actes. L'homme seul est à l'image de Dieu; la femme à la gloire de l'homme (34).

L'homme qui donna un nom aux animaux et qui prêta alors aux choses les vertus de l'agir et du penser donne désormais un nom à sa femme. Cette faculté propre à Adam de donner un nom aux choses est tel un don primitif qui circule et circulera tout au long de l'histoire : ni produite, ni consommée, cette capacité est une valeur dont la seule fonction est l'articulation incessante de la vie et de l'échange. Le nom hébreu hawwâh, forme ancienne de hayyâh, signifie "vivante » et n'est guère éloigné du nom d'Ěve né des Septante et de la Vulgate. Ève, qui signifie «vie », est un signe de contrat, la nécessité interne du développement d'une liberté qui pousse Adam à faire apparaître une médiation ouvrant tous les possibles.

\section{Origines et descendances de Lilith : un visage à plusieurs faces}

La femme obscure qu'est Lilith peut prendre plusieurs visages; ceux de l'antique Circé, de la terrible Gorgone Méduse et de toute femme terrifiante qui séduit ou révulse par ses attraits hors du commun. Lilith est souvent figurée dotée d'une queue de poisson, d'ailes, de nageoires et capable de prendre l'aspect d'un serpent. Fille des mères chtoniennes et terriennes primitives, elle peut être également rapprochée de Mélusine, des ondines et des vouivres, des lamies et des harpies : toutes porteuses d'une séduction dévastatrice.

Le lien de la première Ève juive avec la tradition gréco-latine n'est donc pas à ignorer, même si nous avons tendance à insister sur ceux qu'elle entretient avec la mythologie suméro-babylonienne. Lilith fut en effet souvent rapprochée de Lamia, la reine de Libye abandonnée par Zeus et dont Héra, épouse du dieu, déroba les enfants issus de l'accouplement illégitime. Lamia se vengea de cette cruauté en dévorant les enfants des femmes en couche (ce qui expliquerait certaines morts in utero). Ceci nous rapproche des infanticides commis par Lilith. Une autre croyance caractérise les Lamies comme des créatures démoniaques qui, en séduisant les hommes, leur dévoraient le sang durant l'accouplement. Telles sont les compagnes sym-

(34) Paul. I. COR. XI. 7. 
boliques de notre «adorable » Lilith; elle est donc vraisemblablement issue d'une croyance au carrefour de plusieurs civilisations : un concentré de craintes constitutives d'une unité de l'humain, voire de l'homme. Les caractères telluriques et maritimes qui donnent à la démone l'aspect d'un monstre, d'un batracien ou d'un amphibien, la dévoile comme hybride, aussi présente sur terre que dans l'eau. Elle est également proche des créatures qui ne répondent à aucun lieu naturel spécifique et que le Lévitique qualifie d'animaux impurs et immondes. Elle tire sa monstruosité de son habitat, de son absence de signes susceptibles de la caractériser... peut-être même n'a-t-elle aucun des deux sexes. Ni féminine, ni masculine, elle est un tiers-exclu de la césure naturelle posée entre les sexes. On trouve également dans la mythologie nordique une déesse des eaux, créature ou génie ni mâle, ni femelle, qualifié depuis le XVI ${ }^{\mathrm{e}}$ siècle d'ondine : créature aux aspects aussi innombrables et imperceptibles que les ondes émises sur l'eau par quelque objet ayant touché la surface du liquide. Lilith peut également être représentée parée des attributs qui lui sont déjà assignés : ceux du vent, de la nuit, des forces démoniaques qui troublent les sens et les obscurcissent (35). Le vocabulaire psychanalytique contemporain qualifie d'ondinisme la déviance sexuelle qui consiste à provoquer le plaisir et l'excitation par l'intermédiaire d'un fluide dont le contact et la " texture » suffisent à enivrer et à saouler. Dans la mythologie slave une nymphe des eaux nommée Roussalka hante les rivières et se cache tout en tentant d'attirer à elle les êtres humains de passage pour les « envoûter ». Son aspect n'est pas vaiment celui d'un serpent, mais son caractère aquatique la rend habile dans l'eau et ses jambes lui servent de nageoires (sans être à proprement parler une "femme-serpent», elle réunit les caractéristiques de la Vouivre ou de Mélusine).

Ces exemples empruntés à la mythologie montrent que, par delà leur diversité, ces croyances relèvent de perceptions et de craintes communes, relatives à la figure féminine et aux points d'ombre qui caractérisent sa sexualité et son pouvoir de séduction diabolique, "causes du péché ».

D'un point de vue historique et anthropologique, il serait tentant de considérer toutes ces figures symboliques comme les avatars de l'Ève primordiale oubliée. En cela, le caractère androgyne de ces femmes mythologiques n'est pas à exclure. RÈvenons pour mieux en percevoir la teneur à la tradition judéo-chrétienne de la Genèse et à son lien avec le mythe médiéval de Mélusine. Tout phénomène androgyne relève d'une relation simultanée d'inclusion et d'exclusion entre deux termes : masculin et féminin, ce qui est encore à distinguer de l'hermaphrodisme où le rapport d'inclusion et d'exclusion se fait entre un sexe mâle et un sexe femelle. La "bi-sexualité » organique et psychique de tout 'adâm ne peut pourtant être niée lorsque l'on prend en compte les caractéristiques physiologiques telles que les glandes mammaires rudimentaires chez les hommes et la présence du clitoris chez les femmes qui, très développé à la naissance voit son développement prendre

(35) L'opinion commune de la tradition pense que Lilith resta après son départ de l'Eden dans les eaux de la mer Rouge ; une opinion renforcée par la croyance suivant laquelle l'eau est le plus souvent la demeure des démons. Le vent serait ainsi symbole de la Divinité, le feu celui des Anges et l'eau celui des Démons. On voit bien ici le caractère ambivalent de Lilith, partagée entre les sièges et les forces de la nature. Sur ce point cf. Louis GinZBERG, «La création du monde » in Louis GinZBERG, Les Légendes des Juifs, Paris, Cerf-Institut Alain de Rothschild, 1997, note 40, p. 210 (coll. « Patrimoines »). Se référant au livre 4 de l'Apologia d'ARIstide, Louis GinzBerg insiste sur une "confusion» des croyances entre Lilith, l'esprit du vent babylonien et les Liliths, créatures multiples jugées esprits de la nuit qui sont présents dans le Talmud au Traité Pessahim, 112.a. 
ensuite un retard considérable. L'androgynie est présente dans un grand nombre de représentations collectives, de mythes et de croyances qui conservent la trace d'un être primordial a-sexué. Deux textes de base relevés par Jean Markale (36), celui d'Arras (37) et celui de Couldrette (38), représentent autour de l'an mille quatre cent l'état de la légende de Mélusine dont l'aspect essentiellement chrétien est pourtant d'une stricte orthodoxie. Mélusine, souvent associée aux puissances nocturnes, fut à la suite d'un combat entre principe féminin et principe masculin (rencontre de la lune et du soleil) déterminée au lieu du sommeil de la raison: l'Obscurité ; elle doit se cacher des hommes le samedi, sa queue de serpent faisant foi de son caractère hybride et monstrueux. Parce que le sommeil de la raison engendre des monstres, Mélusine trouve en celui-ci son procréateur et son lieu d'existence. Le tentateur anguipède, thème cher à la Genèse, se retrouve ici encore sous la forme d'un corps amphibien associé à une tête et à un buste humain : celui d'une femme séduisante en tous points, mais qui garde en elle le secret de sa nature (homme par les attributs qu'elle cache, femme par ceux qu'elle peut montrer, sa queue de serpent lui donnant les attributs d'une femme phallique qui dépasse l'humain grâce à l'association des deux genres sexués. Il s'agit une fois de plus ici d'un être unifié réunissant en son sein des forces opposées (39). Notons que de nombreuses divinités agricoles grecques portent cette même trace d'androgynie. Chez les Estoniens, les divinités agricoles réputées mâles une année peuvent être reconnues femelles l'année suivante. La double caractéristique du corps de Mélusine manifeste également du scindé dans du non-séparé et la présence d'une force centripète attirant à elle tous les contraires pour les faire siens dans l'unité. Mélusine appartient à la terre : sa queue de serpent fait foi de son caractère tellurique. Cette prégnance du symbole de la terre n'est pas sans rappeler notre récit où Adam et Lilith sont tirés de la Terre, du limon pour l'un, et suivant les versions, d'immondices pour l'autre.

Ève et Lilith, doubles figures d'une même femme primordiale, font indéniablement penser aux deux Aphrodites du Banquet de Platon. Qu'il s'agisse de l'Aphrodite céleste Ourania, fille d'Ouranos n'ayant pas de mère et ne procédant que du principe masculin, ou de l'Aphrodite Pandémos, fille de Zeus et de Dioné, nous retrouvons cette même image : celle de deux puissances féminines opposées dont l'une tente de ramener à elle tous les principes qui lui sont étrangers pour se les approprier. L'analogie entre les deux Aphrodites et les deux figures de la femme primordiale n'est pas dénuée de fondement. Rappelons en effet que l'Aphrodite pandémienne a un temple dans lequel Solon posa la réglementation de la prostitution à Athènes. Cette Aphrodite dont, selon Platon, l'amour s'adresse aux hommes comme aux femmes, semble s'attacher à notre Lilith. Des Gorgones à Mélusine via Lilith, toutes ces créatures aux caractéristiques amphibiennes et dont l'usage des plaisirs n'est pas la génération, inspirent des analogies lourdes de sens.

\section{Vanessa ROUSSEAU Université de Paris XIII-Villetaneuse}

(36) Jean Marlale, Mélusine, Paris, Éditions Albin Michel, 1993 (coll. « Espaces libres »).

(37) Jean d'Arras, Mélusine, Paris, Éditions Stock, 1991 (coll. « Moyen Âge »).

(38) Couldrette, Le Roman de Mélusine, Paris, Garnier Flammarion, 1993.

(39) Telle est la manifestation d'une unité primordiale des opposés, cf. Mircea Eliade et son concept de coüncidentia oppositorum, in Méphistophélès et le mythe androgyne, Paris, Gallimard, 1962 (coll. "Idées »). 
Résumé

Qui est donc Lilith, cette figure féminine qui dans la tradition juive est réputée avoir été la première compagne d'Adam?

En partie oubliée par l'histoire et les textes d'exégèses issus de la chrétienté, Lilith prit une place sporadique et trouble dans les différents systèmes de croyances judéo-chrétiens portés sur la Femme et sa sexualité. Cette démone est au carrefour de plusieurs religions et demeure, semble $t$-il, la figure féminine qui témoigne le plus universellement des craintes, préjugés et désirs portés sur la Femme et ses mystères supposés. On la retrouve au centre de plusieurs mythes hérités de populations diverses, ce qui pourrait témoigner d'une structure unitaire du penser humain concernant le féminin. Les récits hébraïques et juifs la présentèrent sous l'aspect d'une démone aux attributs masculins et dévorateurs. Malgré cette présence dans la littérature juive, on ne la trouve guère présente dans les écrits de la chrétienté antérieurs au XVI siècle, si ce n'est dans un texte de saint Augustin qui la déclare être une illusion de l'esprit. Les versions de la Bible et conceptions chrétiennes ne gardèrent pour figure centrale du mythe de la création qu'une seule femme: l'Ève que nous connaissons. Selon les croyances, Lilith fut remplacée par cette femme plus sage. Il faut attendre la Renaissance pour trouver le nom de Lilith dans les écrits chrétiens. Son caractère androgyne la place au centre de tous les mythes qui traitent de la sexualité, de l'amour, de la distinction des sexes, de la question des origines, du pouvoir et de la force la plus obscure de l'humain: son animalité.

\section{Abstract}

Who is Lilith, this feminine figure who, according to Jewish tradition, is supposed to have been Adam's first companion?

More or less forgotten by history and Christian exegetical texts, Lilith holds a sporadic and somewhat vague position in the various Judeo-Christian belief systems related to women and feminine sexuality. This demon is at the crossroads of several religions and seems to be the feminine figure that most universally represents the fears, prejudices and desires directed towards women and their supposed mysteries. She appears at the center of several myths that have come down from populations as diverse as they are distinct, which could suggest a certain universalism in the structure of human thought concerning the feminine. Lilith came into existence in the form of Hebraic and Jewish accounts, being presented in Talmudic and Midrashic literatures as a devouring female demon with masculine traits. Despite this presence in Jewish literature, she is barely present in Christian writings before the $16^{\text {th }}$ century, with one notable exception in a text by Saint Augustine, who declares her to be an illusion of the mind. The various versions of the Bible and Christian conceptions retained only one woman in the central myth of the creation: Eve. According to some beliefs, Lilith was replaced by this more wellbehaved woman. It is not until the Renaissance that one finds the name Lilith in Christian writings. Lilith's androgynous character places her at the center of all myths that deal with sexuality, love, the distinction between the sexes, the question of origins, power and the most obscure force in humanity: its animal nature.

\section{Resumen}

Quien es Lilith, esta figura femenina que en la tradición judía es supuestamente la primera compañera de Adán?

En parte olvidada por la historia y los textos de exégesis originarios de la cristiandad, Lilith toma un lugar esporádico y poco claro en los diferentes sistemas de creencias judeo-cristianas sobre la Mujer y su sexualidad. Este demonio está en la encrucijada de varias religiones, y permanece aparentemente como la figura femenina que testimonia el más universal de los temores, prejuicios y deseos sobre la Mujer y sus supuestos misterios. Se la encuentra en el centro de muchos mitos heredados de poblaciones diversas, lo que podría testimoniar la existencia de una estructura unita- 
ria del pensamiento humano relativo a lo femenino. Los relatos hebraicos y judios, la presentan bajo el aspecto de un demonio de atributos masculinos y devoradores en las literaturas talmúdicas y midráquicas. Aunque presente en la literatura judía, no se la encuentra presente en los escritos de la cristiandad anteriores al siglo XVI, excepto en un texto de San Agustín, que la declara una ilusión del espíritu. Las versiones de la Biblia y las concepciones cristianas conservaron como figura central del mito de la creación a una sola mujer: la Eva que nosotros conocemos. Según las creencias, Lilith fue reemplazada por esta mujer más prudente. Hay que esperar al Renacimiento para encontrar el nombre de Lilith en los escritos cristianos. Su carácter andrógino la ubica en el centro de todos los mitos que tratan sobre la sexualidad, el amor, la distinción de los sexos, la cuestión de los orígenes, del poder y de la fuerza más oscura del ser humano: su animalidad. 
